\title{
Swiss Insurance Medicine SIM
}

Auf Initiative von an Versicherungsmedizin speziell interessierten Kollegen aus den Reihen der Schweizerischen Gesellschaft für Versicherungspsychiatrie (SGVP), der Vertrauensärzte (SGV), der Schweizerischen Gesellschaft für Traumatologie und Versicherungsmedizin (SGTV), der Association Romande des Praticiens en Expertises Médicales (ARPEM), des SVV, der Suva, IV, MV sowie der Neurologengesellschaft und der FMH wurde Anfang September die Swiss Insurance Medicine (SIM, Interessengemeinschaft Versicherungsmedizin) aus der Taufe gehoben. Sie hat sich mehrere Ziele auf ihr Banner geheftet: Erstellen eines Bildungskataloges mit versicherungsmedizinrelevanter Aus-, Weiter- und Fortbildung, mittelfristig das Anstreben eines Fähigkeitsausweises Versicherungsmedizin und langfristig eine Akademie für Versicherungsmedizin. Daneben sind projektbezogene Aktivitäten geplant.

Im Festvortrag anlässlich der Gründungsversammlung konnte uns Erwin Murer, ordentlicher Professor für Arbeits- und Sozialversicherungsrecht an der Universität Freiburg i. Ü., sehr aktuelle Gedanken nahebringen zu einem Thema, das von einzelnen Parteien für den Wahlkampf für National- und Ständerat gepachtet wurde und dadurch eine erhebliche Medienpräsenz aufweist. Zum gleichen Thema ist in der NZZ am Sonntag vom 21. September 2003 ein Interview mit Prof. Murer erschienen. Er äussert dort sehr interessante Gedanken, welche mithelfen, das Problem der zunehmenden IV-Renten etwas differenzierter zu betrachten.

Im in dieser Ausgabe der Schweizerischen Ärztezeitung abgedruckten Referat (S. 2312-14) macht Prof. Murer einige besonders bedenkenswerte Aussagen. Die wohl bedeutsamste ist die
Forderung nach Interdisziplinarität zwischen Jurisprudenz und Medizin. Dies klingt zwar nach einer Selbstverständlichkeit. Ich zitiere in diesem Zusammenhang aus dem Geleitwort von Prof. Murer, das er für die demnächst erscheinende 2. Auflage des «Manuals der Schweizer Vertrauensärzte» geschrieben hat:

«Fast ebenso wichtig ist dabei die Wahrnehmung der unterschiedlichen Denkweisen von Medizinern und Rechtsanwendern. Wie viele Missverständnisse könnten in der Zusammenarbeit vermieden werden, wenn sich die Beteiligten über diesen wichtigen Unterschied mehr Rechenschaft geben würden! So hat der Rechtsanwender beispielsweise die Tendenz, vom Mediziner ein klares Ja oder ein klares Nein zu erwarten. Denn genau das verlangt von ihm meistens auch die Rechtsnorm, die er anwenden muss: Entweder liegt 〈Krankheit im Sinne des KVG vor, oder sie liegt eben nicht vor - tertium non datur. Anders die Sichtweise des Mediziners; für ihn gibt es vielleicht zehn Möglichkeiten zwischen 〈Kranksein〉 und «Nicht-mehr-krankSein`! Tatsächlich ist im Zusammenwirken zwischen Medizin und Recht echte Interdisziplinarität angesagt - und es gibt wohl niemanden, der dermassen eng an der Schnittstelle zwischen den beiden Wissenschaften positioniert ist wie der Versicherungsmediziner.»

Dem ist nichts mehr anzufügen ausser dem Wunsch, dass das in dieser Ausgabe abgedruckte Murer-Referat viele interessierte Leser findet.

Dr. med. Jürg Zollikofer, Präsident der SGV und der SIM, Ossingen 